\title{
EFICÁCIA PARA O COMBATE DA BROCA DA CANA-DE-AÇÚCAR
}

\author{
EFFECTIVENESS FOR COMBATING SUGAR CANE DRIL
}

\author{
Jéssica de Assis Gomes - jessicaassis09@hotmail.com \\ André Luiz Oliveira - andre.adv@uol.com.br \\ Faculdade de Tecnologia de Taquaritinga (Fatec) - Taquaritinga - São Paulo - Brasil
}

DOI: 10.31510/infa.v17i1.818

RESUMO

O objetivo desse artigo é demonstrar como a broca-da-cana age na cana-de-açúcar, abordando também como seu inimigo natural a Cotesia flavipes combate essa praga. Através de pesquisas bibliográficas, foi apresentado como o método natural é eficaz para combater a praga da cana, os seus benefícios e a importância desse método para o meio ambiente, já que o país é marcado por forte presença de agrotóxicos no setor do agronegócio. Também foi descrito como ele foi introduzido no Brasil, a história do controle biológico e o seu crescimento com o tempo. A pesquisa contemplou ainda a importância da cana-de-açúcar para a economia do país e a razão pela qual o controle biológico da broca-da-cana cresceu em larga escala no país, perpassando por temas como a nocividade dos agrotóxicos para a saúde humana, além de prejuízos de contaminação de vários ecossistemas, especialmente solos e rios. Por fim, o estudo demonstrou como é feito o controle e a produção da broca-da-cana e da Cotesia flavipes em laboratório de entomologia, desde a identificação de seus ciclos e a maneira como ocorre a liberação da vespa no campo.

Palavras-chave: Entomologia. Pragas. Controle Biológico. Método Natural.

\begin{abstract}
The objective of this article is to demonstrate how the sugarcane drill acts in sugarcane, also addressing as its natural enemy the Cotesia flavipes fights this plague. Bibliographical research has presented how the natural method is effective in combating the sugarcane pest, its benefits, and the importance of this method for the environment, given that the country is marked by a strong presence of agrochemicals in the agribusiness sector. It has also been described how it was introduced in Brazil, the history of biological control and its growth over time. The research also contemplated the importance of sugarcane for the country's economy and the reason why biological control of the sugarcane drill has grown on a large scale in the country, going through topics such as the harmfulness of agrochemicals to human health, as well as the damage caused by contamination of various ecosystems, especially soils and rivers. Finally, the study demonstrated how the control and production of the sugarcane borer and the Cotesia flavipes is done in an entomology laboratory, from the identification of its cycles and the way the wasp is released in the field.
\end{abstract}

Keywords: Entomology. Pests. Biological Control. Natural Method. 


\section{INTRODUÇÃO}

A cana-de-açúcar é uma das principais culturas do mundo, sendo cultivada por mais de 100 países, sendo que a mão de obra rural nesses países tem uma importante representação. Ela é uma planta nativa do sul e sudeste asiático, mas no continente europeu seu maior cultivo foi na Espanha. No continente americano os países que tiveram seus cultivos foram Brasil, Cuba, México, Peru, Equador, Colômbia e Venezuela.

O Brasil é o maior produtor de cana-de-açúcar do mundo, e o estado de São Paulo é onde fica a maior produção do país, onde corresponde a 55\% da área plantada no país. De acordo com União da Indústria de Cana-de-Açúcar, as 172 usinas instaladas no estado correspondem a $42 \%$ do total brasileiro e foram responsáveis por $56 \%$ da cana moída nacionalmente na Safra 2016/2017. O Estado de São Paulo é destaque no uso de energia de fontes renováveis, a participação dessas fontes na matriz energética paulista chegou a $60,8 \%$ (SECRETARIA DE ENERGIA, 2016).

A cana-de-açúcar é um dos maiores meios de economia do país, por isso é preciso que sua produção caminhe junto com o meio ambiente. A cana é um dos principais alvos de pragas, cuja sua produção acaba sendo prejudicada e a sua qualidade fica comprometida, pois dependendo da infestação da praga a empresa acaba sendo prejudicada economicamente.

Uma das pragas que mais prejudica a cana-de-açúcar é conhecida como broca-da-cana (Diatraea Saccharalis), a sua ação pode causar fungos e pragas secundárias, com o pensamento cada vez maior da preservação do meio ambiente, são necessárias pesquisas de qual método natural mais eficaz para combatê-las. Com o crescimento da produção da cana fica mais difícil usar os métodos químicos, pois além de prejudicar a saúde do trabalhador, tem a contaminação do solo que prejudica tanto os organismos terrestres e aquáticos, sendo assim o controle biológico se torna o método mais favorável, mesmo que sua ação seja mais lenta. (EMBRAPA, 2016)

Segundo Zucchi et al. (1993) essa praga ataca principalmente a cana-de-açúcar, trigo, capins, arroz, aveia, milho e sorgo. A broca-da-cana pode ser encontrada o ano todo, com o seu auge sendo atingido em setembro e pico populacional em janeiro.

O objetivo desse trabalho é demostrar as vantagens que se tem ao usar o método natural para combater a broca da cana, evitando agressões severas ao meio ambiente em caso de 


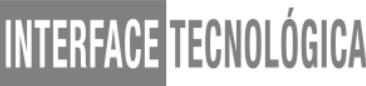

utilização de agrotóxico, evidenciando ainda como o método biológico é menos dispendioso em relação ao defensivo agrícola.

\section{FUNDAMENTAÇÃO TEÓRICA}

\subsection{Cana-de-Açúcar}

No Brasil umas das principais fontes de economia é a cana-de-açúcar: com ela é produzido o etanol e o açúcar, produtos que movimentam a economia mundial. As usinas sucroalcooleiras utilizam toda a cana, depois de produzir o açúcar e o álcool elas usam o que sobra para gerar energia. Essas usinas geralmente ficam localizadas junto às regiões produtoras de cana. Desta forma, o Estado de São Paulo se destaca por apresentar boas condições de solo e clima, ter uma infraestrutura de transportes adequada, proximidade dos mercados consumidores e uma grande base de desenvolvimento científico e tecnológico (BNDES, 2008).

Um dos maiores incentivos para a utilização e venda do etanol como um biocombustível desde a década de 1970 até os dias de hoje é a preocupação da sociedade com as grandes emissões caudadas pelos veículos de uma forma excessiva, e com a qualidade do ar nas grandes cidades (LEITE; LEAL, 2007).

Para Macedo (2007) a busca constante da indústria nacional de acompanhar as transformações mundiais dos produtos derivados da cana-de-açúcar, o ritmo das exportações, assim como as crises financeiras pelas quais passaram os países em desenvolvimento antes da adoção de políticas econômicas neoliberais levaram o Brasil ao movimento de adotar grandes programas de incentivo em relação à produção canavieira, sempre visualizando os avanços tecnológicos e a mecanização dos processos ao longo da história recente das grandes indústrias e usinas nacionais.

As inovações fundamentais para as tecnológicas no sistema agroindustrial da cana-deaçúcar são automação, melhoramento genético, monitoramento por satélite, fermentação contínua e lançamento de novos produtos (FERREIRA et al., 1985). De acordo com Dunham et al. (2010), a estratégia pautada para melhorar e direcionar a pesquisa agrícola no Brasil foi a seleção de variedades de cana-de-açúcar existentes no país e no exterior. O desenvolvimento e seleção de variedades de cana têm como objetivo aumentar a produtividade agroindustrial visando maior concentração de sacarose e resistência a pragas e doenças. 


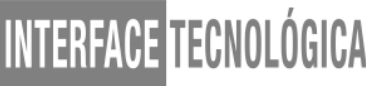

Uma das melhorias em relação às tecnologias utilizadas é a colheita mecanizada da cana-de-açúcar, que também ajuda na eliminação de ervas daninhas do canavial. A palha mantida sobre o solo impede a germinação e o estabelecimento de espécies com poucas reservas armazenadas na semente, o que diminui a dependência de herbicidas. A cobertura do solo pela palha traz muitos outros benefícios para o meio ambiente, saúde da população e aspectos econômicos, por outro lado, a manutenção da palha no cultivo ocasiona o aumento de pragas, especialmente a broca-da-cana (Diatraea saccharalis) (FERREIRA et al., 2010; SANTIAGO; ROSSETTO, 2016).

\subsection{Broca-da-Cana}

Dentre as pragas existentes no setor do agronegócio brasileiro, a broca-da-cana é uma das pragas mais relevantes do ponto de vista da potencialidade de gerar prejuízos. O adulto é uma mariposa de hábitos noturnos, realizando a postura na parte dorsal das folhas, sendo a razão principal da redução significativa da produtividade no campo e a qualidade da matéria-prima.

Os danos causados pela broca ocorrem durante todo o ciclo da cultura e podem causar menor produtividade agrícola, pois os colmos perdem peso, são menores e mais finos muitos secam e morrem, outros se quebram pela ação do vento, já que estão mais frágeis devido às galerias em seu interior; quando o ataque se dá próximo à região de crescimento, ocorre morte da gema apical, com brotação das gemas laterais e consequentemente, inversão de sacarose (Dinardo-Miranda, 2008).

Com base em Nakano et al. (2002), constatou-se que após 4 a 9 dias da postura, as larvas recém eclodidas, passam a se alimentar do parênquima das folhas. As lagartinhas descem pela folha penetrando no colmo, perfurando-o na região dorsal. No interior do colmo cavam galerias permanecendo até o estágio adulto. Após cerca de 40 dias, as lagartas com 2,2 a 2,5 cm, abrem um orifício, fechando-o com fios de seda e serragem. Nessa fase, transformam-se em mariposas, saindo pelo orifício aberto e atingem novas plantas.

Conforme Pinto (2006), o monitoramento da população da praga, realizado por meio de levantamentos da quantidade de lagartas, serve para definir o momento certo para a opção de uma medida de controle. Esse monitoramento é feito durante a fase vegetativa da cana, até sua maturidade. A estimativa de danos é realizada no momento da colheita, na frente de corte, ou na chegada da cana na usina, servindo para identificar as áreas-problema que deverão ser monitoradas na safra seguinte. 


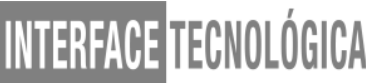

Segundo Salvadori e Parra (1990), um dos primeiros passos a serem feitos para a realização de estudos biológicos e desenvolvimento de métodos de controle de um inseto é a definição de uma dieta de artificial que permita a sua criação, integrando requisitos mínimos de qualidade biológica.

\subsection{Malefícios da Utilização do Agrotóxico}

Segundo Spadotto (2006), a utilização de agrotóxicos na cana-de-açúcar, assim como em qualquer cultura traz várias consequências para o meio ambiente e para a população, o uso frequente e incorreto pode causar contaminação de solos, das águas superficiais e subterrâneas, dos alimentos, da atmosfera. Com o uso excessivo de agrotóxico pode elevar os efeitos negativos em organismos terrestres e aquáticos, como: a intoxicação humana pelo consumo de água e alimentos contaminados e intoxicação ocupacional de trabalhadores e produtores rurais.

É muito importante que os produtores levem em conta o comportamento ambiental dos agrotóxicos, pois eles em geral, tem uma classificação de comportamento ambiental relacionadas a periculosidade. Por isso é indispensável avaliar o modo como conciliar a demanda de produtos originados da cana-de-açúcar, com viabilidade econômica e feitios como a saúde e o meio ambiente (SOUZA; MIZIARA, 2010). Por essa razão defende-se o uso do método do controle biológico, pois não afeta o meio ambiente e nem a saúde da população.

\subsection{Controle Biológico}

O controle biológico de pragas é um método natural em que um organismo vivo, denominado com inimigo natural, combate a população de outro organismo, a praga. A praga mais conhecida da cana e a mais importante economicamente para o estado de São Paulo é a broca-da-cana, cujo controle demanda uma análise multivariada, que envolve conhecimentos quanto à biologia do agente causador, sobre os níveis de danos provocados e as consequências econômicas decorrentes, o custo do controle e os impactos que os métodos de controle podem causar. Atualmente, o controle mais eficiente de Diatraea saccharalis é o biológico, por meio do uso do parasitoide de larvas Cotesia flavipes e do parasitoide de ovos Trichogramma galloi (MACEDO; MACEDO, 2004; NAVA et al., 2009).

Os benefícios da utilização do controle biológico no cultivo de cana são vários, e ressalta-se que esse método busca atingir somente o organismo alvo de controle. Conforme Oliveira (2018) no controle biológico, somente o alvo é combatido, com isso o risco ambiental é mínimo, não deixando resíduos tóxicos em alimentos, água e solo, além disso o uso do 
controle biológico tem ausência de período de carência entre a liberação do inimigo natural e a colheita, o controle biológico também é caracterizado por não provocar o surgimento de populações de pragas resistentes, além de não afetar outros métodos de controle, nem oferecer perigo a saúde humana.

A vespa Cotesia flavipes foi introduzida no Brasil para controlar biologicamente o hospedeiro Diatraea saccharalis, conhecida como a broca da cana-de-açúcar, ela é o grande inimigo natural da broca (UEHARA, 2007). De acordo com Parra (2000) os parasitoides produzidos em laboratório são os mais utilizados no controle de pragas, devido ao baixo custo, fácil manipulação e por se alimentarem da própria praga, não tendo necessidade de uso de uma dieta específica para eles.

Segundo Santos (2009) para produzir os inimigos naturais da broca da cana-de-açúcar em laboratório, é preciso ter um conhecimento avançado de entomologia, conhecer todas as características da praga e do agente entomopatogênico, sendo necessário produzir primeiramente a própria praga.

Conforme Mena (2010) existem duas formas de controle da broca da cana-de-açúcar, o controle biológico ou o controle químico. No controle químico são usados inseticidas de diferentes modos de ação assim como diferentes princípios ativos, ao passo que no controle biológico utiliza-se do parasitoide larval, a Cotesia Flavipes, pois é um dos inimigos mais utilizados em cana-de-açúcar com eficiente controle em condições de campo.

Quando se escolhe realizar o controle através da forma química, para que se tenha eficiência de inseticidas, esses devem ser aplicados enquanto as lagartas são ainda jovens e antes das mesmas penetrarem no interior do colmo, já o uso da Cotesia Flavipes para controle de broca em cana é um dos maiores programas de controle biológico em todo o mundo, com base na extensão da área tratada e sua alta eficácia (VACARI; DE BORTOLI, 2010).

\section{PROCEDIMENTOS METODOLÓGICOS}

A presente pesquisa possui caráter descritivo e exploratório e procura evidenciar uma questão totalmente voltada para um dos principais desafios da humanidade: a conciliação entre formas sustentáveis de produção e mitigação de danos decorrentes do uso de agrotóxicos, como forma de mitigar os danos ambientais oriundos dos prejuízos que esses agentes causam à saúde das populações. 
De acordo com Gil (2006), o objetivo de uma pesquisa exploratória é compreender um assunto ainda pouco estudado, abordando uma situação, entidade ou conjunto de entidades que têm um mesmo comportamento ou são do mesmo perfil.

Esse trabalho teve o desenvolvimento a partir de pesquisas bibliográficas, com coleta de dados em artigos científicos, literatura específica sobre o tema, buscando evidenciar os benefícios do controle biológico em detrimento à utilização de métodos químicos.

\section{RESULTADOS E DISCUÇÃO}

O Controle Biológico teve uma grande dificuldade de ser implantado no Brasil, pois a cultura que predominava era o uso de agentes químicos, pois desde sempre a maior tradição do país foi no sentido de se combater qualquer praga quimicamente, daí a resistência do controle biológico ser implantado. Segundo Parra (2017) o Brasil é o país que mais usa produtos químicos no mundo no meio agrícola, pois nossos agricultores têm como forte característica essa cultura.

Os países da América Latina têm mais tradição de controle biológico do que o Brasil, que tinham muita resistência em adotar essa cultura pois os agricultores sempre preferiam um método que acabasse de forma mais rápida com as pragas da cana, e não explorava a sua rica biodiversidade existente. Conforme Parra (2017) com o tempo os agricultores começaram a aceitar mais a ideia de utilizar o controle biológico aplicado, pois como é uma liberação em massa dos predadores os efeitos se assemelham mais aos dos inseticidas.

A maior dificuldade que se tinha em implantar o Controle Biológico é o grande território existente no país, pois o monitoramento de pragas e liberação dela requer muita atenção. O período que o Brasil mais teve dificuldade foi do ano de 1940 até o ano de 1960, após esse período difícil teve o surgimento do MIP (Manejo Integrado de Pragas), foi uma resposta da comunidade cientifica para mostrar como usar de forma adequada os defensivos, como controlar as pragas de uma forma que contribuísse com os aspectos econômicos, juntamente com o lado social e ecológico.(Parra, 2017)

Segundo os dados do Ministério da Agricultura esse tipo de controle cresceu 652\% no ano de 2017, e esse aumento tem como principal fator a população, que tem como um objetivo maior alimentos livres de agrotóxicos, e com isso o Brasil segue cada vez mais avançando e melhorando o seu Controle Biológico. 


\subsection{Controle e Produção da Broca-da-Cana}

O controle da broca-da-cana é feito através dos seus inimigos naturais, sendo que um dos principais agentes neste sentido é a Cotesia flavipes. Para poder fazer esse controle biológico é necessário criar um ciclo que combate essa praga, ciclo este que é criado através de produção em laboratório, mas é preciso ter um grande conhecimento sobre entomologia para poder conhecer todo o processo necessário. (SANTOS, 2009)

Por se tratar de seres vivos é importante saber como cada praga funciona, bem como suas características, como reagem e qual a melhor maneira de cria-las. O laboratório de entomologia deve ser o mais adequado possível, ser limpo e que tenha salas adequadas para cada procedimento. Para o início do ciclo é preciso criar a praga, pois é a partir dela que se tem toda a produção necessária para combater a broca-da-cana no campo.

De acordo com Parra (2008) para a produção da praga é necessária uma dieta artificial que tenha tudo o que a broca-da-cana necessita para se desenvolver de uma forma saudável, e uma dieta de realimentação que mantenha os nutrientes até a liberação da vespa.

A tabela 1 contempla os ingredientes para se fazer a dieta artificial da praga e as quantidades necessárias para ter uma produção.

Figura 1: Tabela de Dieta de Alimentação e Realimentação da Diatraea

Saccharalis.

\begin{tabular}{|c|c|c|c|c|c|c|c|}
\hline \multirow{3}{*}{$\begin{array}{l}\text { Ingredientes } \\
\text { Água destilada }\end{array}$} & \multicolumn{3}{|c|}{ Dieta de alimentaçāo } & \multicolumn{4}{|c|}{ Dieta de realimentaçāo } \\
\hline & \multicolumn{2}{|c|}{ Sem levedura } & Com levedura & \multicolumn{2}{|c|}{ Sem levedura } & \multicolumn{2}{|c|}{ Com levedura } \\
\hline & 12 & $\mathrm{~L}$ & $12 \mathrm{~L}$ & $11 \mathrm{~L} 400$ & $\mathrm{~mL}$ & $11 \mathrm{~L} 400$ & $\mathrm{~mL}$ \\
\hline Açúcar cristal & 130 & g & $130 \mathrm{~g}$ & 540 & g & 540 & g \\
\hline Farelo de soja & 900 & g & $900 \mathrm{~g}$ & 800 & g & 800 & $g$ \\
\hline Germe de trigo & 600 & $g$ & $400 \mathrm{~g}$ & 350 & $\mathrm{~g}$ & 260 & $g$ \\
\hline Nipagin & 80 & $g$ & $80 \mathrm{~g}$ & 80 & g & 80 & $\mathrm{~g}$ \\
\hline Ácido ascórbico & 24 & $g$ & $24 \mathrm{~g}$ & 10 & $\mathrm{~g}$ & 10 & $\mathrm{~g}$ \\
\hline Levedura & - & & $800 \mathrm{~g}$ & - & & 460 & $\mathrm{~g}$ \\
\hline Sais de Wesson & 40 & $g$ & $40 \mathrm{~g}$ & - & & - & \\
\hline Cloreto de colina & 4 & $\mathrm{~g}$ & $4 \mathrm{~g}$ & 4 & $g$ & 4 & $g$ \\
\hline Soluçāo vitamínica & 160 & $\mathrm{~mL}$ & $160 \mathrm{~mL}$ & 80 & $\mathrm{~mL}$ & 80 & $\mathrm{~mL}$ \\
\hline Formol & 26 & $\mathrm{~mL}$ & $26 \mathrm{~mL}$ & 10 & $\mathrm{~mL}$ & 20 & $\mathrm{~mL}$ \\
\hline Vita gold & 60 & $\mathrm{~mL}$ & $60 \mathrm{~mL}$ & 4 & $\mathrm{~mL}$ & 4 & $\mathrm{~mL}$ \\
\hline Ampicilina & 1 & comp & 1 & 2 & comp & 2 & \\
\hline Wintomylon & 10 & $\mathrm{~mL}$ & $10 \mathrm{~mL}$ & 10 & $\mathrm{~mL}$ & & \\
\hline Caragenato & 220 & $\mathrm{~g}$ & $220 \mathrm{~g}$ & 280 & $g$ & 280 & $\mathrm{~g}$ \\
\hline Ácido acético & - & & - & 80 & $\mathrm{~mL}$ & 80 & $\mathrm{~mL}$ \\
\hline
\end{tabular}

Fonte: SCIELO (2012) 


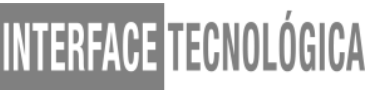

Depois de feita a produção da Diatraea Saccharalis, começa a produção da Cotesia Flavipes. Para ter uma boa produção é preciso que a praga esteja sem defeito, pois ela servirá como hospedeira da Cotesia flavipes. A produção da vespa requer muito cuidado, é necessário que a broca seja manuseada com cuidado para não gerar lesões, sendo importante frisar que somente a fêmea da vespa que deposita os ovos.

Na figura 2 observa-se o ciclo da broca até chegar ao ciclo de lagarta, onde se inocula a praga, e o ciclo da Cotesia flavipes, até a sua liberação no campo.

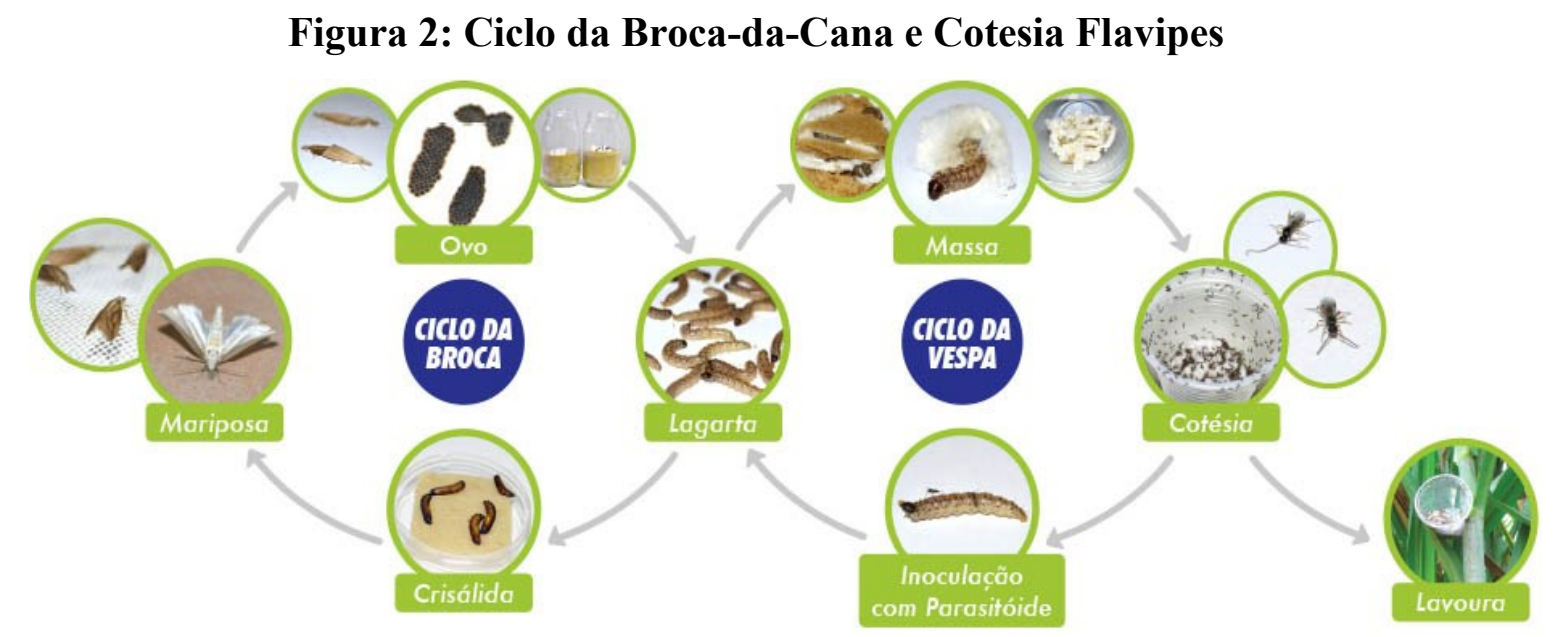

Fonte: Gales Machado (2018)

Após o ciclo completo da Cotesia flavipes é feita a liberação no campo, onde serão coletados os dados para saber se a vespa obteve eficácia ao combate da praga.

\subsection{Liberação da Cotesia Flavipes e seus Resultados}

Para fazer a liberação da Cotesia flavipes é necessário um controle das áreas infestadas, sendo que a liberação da vespa é feita na parte da manhã. Como possui apenas 120 horas de vida, é importante que ela seja liberada nas suas primeiras 24 horas, como forma de obtenção de um resultado mais eficaz.

A liberação da vespa no campo tem que ser a 25 metros do início do talhão, coloca-se os copos onde fica a Cotesia flavipes sem tampa nas folhas da cana, conta-se mais 50 metros e coloca outro copo, assim é feita a liberação da vespa em toda a área que está infectada da brocada-cana. É feita uma avaliação após 10 a 15 dias da liberação, é preciso retornar ao campo e coletar as lagartas para ver se houve o parasitismo e se houve eficiência no processo. 
A Cotesia flavipes tem mais eficácia do que o agrotóxico por elas conseguirem inserção dentro da cana (onde a broca se localiza), já que a vespa é capaz de sentir o cheiro da praga, porque é um cheiro atrativo para a Cotesia flavipes. Além disso o método de controle biológico é mais barato do que o uso de inseticidas, o custo da Cotesia flavipes é de $\mathrm{R} \$ 17$ por hectare, incluindo produto, mão-de-obra e transporte, mesmo sua ação sendo mais lenta, ela consegue ser mais eficiente (ALEXANDRE PINTO, 2010).

\section{CONSIDERAÇÕES FINAIS}

Este trabalho evidenciou a importância do Controle Biológico na cultura da cana-deaçúcar, como alternativa mais adequada à utilização de agrotóxicos, tendo em vista sua potencialidade de gerar danos à saúde humana e ecossistemas.

Constatou-se que, para implantar o controle biológico no Brasil, foi preciso muito estudo para esse método se consolidasse no país, ante grande resistência dos agricultores, que optavam por métodos mais rápidos para exterminar as pragas da cana-de-açúcar.

Após pesquisas científicas sobre o tema, foi possível identificar e se estabelecer uma melhor compreensão acerca de quais métodos são os melhores para a liberação da Cotesia flavipes, o inimigo natural da cana, desde sua criação e seu método de ação como controle biológico natural, constatando-se que a vespa é o vetor mais eficaz no combate à broca.

Para que esse tipo de cultura fosse forte na agricultura brasileira, foi necessária muita pesquisa, saber como montar um laboratório biológico, pesquisar as características da praga e de seu inimigo e montar uma dieta artificial para criar uma produção grande para o campo.

Por fim, é possível concluir que que apesar do resultado da Cotesia flavipes ser mais lenta em relação ao uso de inseticida, este método se mostrou mais eficaz, sendo um dos motivos para a abertura do controle biológico no Brasil, além da preocupação em relação a saúde da população e a preocupação ao meio ambiente. Sob essa perspectiva é importante a recomendação no sentido de que os laboratórios entomológicos aprimorem cada vez mais suas técnicas e pesquisas visando estudos continuados e assertivos com relação aos métodos de evolução do Controle Biológico no Brasil. 


\section{REFERÊNCIAS}

ABCUSTOS. ANÁlise DOS CUSTOS DE PRODUÇÃO DOS AGENTES BIOLÓGICOS PARA CONTROLE DE DIATRAEA SACCHARALIS EM CANA-DEAÇÚCAR: UM ESTUDO DE CASO EM LABORATÓRIO DE PRODUÇÃO MASSAL. Disponível em: <https://abcustos.emnuvens.com.br/abcustos/article/view/282>. Acesso em: 01 jun. 2019.

AGRISHOW. Benefícios do controle biológico no cultivo de cana no Brasil. Disponível em: $<$ https://digital.agrishow.com.br/beneficios-do-controle-biologico-no-cultivo-de-cana-nobrasil/>. Acesso em: 30 mai. 2019.

CANAL6. Cana-de-açúcar e seus impactos: uma visão acadêmica. Disponível em: $<$ http://www.canal6.com.br/livros_loja/Ebook_Cana.pdf>. Acesso em: 28 mai. 2019.

CENTRO DE TECNOLOGIA CANAVIEIRA. Pragas e Doenças da Cana-de-Açúcar. Disponível em: $<$ https://variedadesctc.com.br/wp-content/uploads/2018/07/Caderneta-dePragas-e-Doenças-da-Cana-de-açúcar-CTC.pdf>. Acesso em: 31 mai. 2019.

DIA DE CAMPO. Controle biológico da cana no Brasil é o maior do mundo. Disponível em: http://www.diadecampo.com.br/zpublisher/materias/Newsletter.asp?id=21595\&secao=Pacotes \%20Tecnol\%F3gicos. Acesso em: 30 ago. 2019.

EXAME. Uso do controle biológico de pragas dispara no Brasil. Disponível em: https://exame.abril.com.br/ciencia/uso-do-controle-biologico-dispara-no-brasil/. Acesso em: 24 ago. 2019.

G.BIO. Danos causados pela broca-da-cana, Diatraea saccharalis (Fabr.) (Lepidoptera: Crambidae), em cinco variedades de cana-de-açúcar. Disponível em: $<$ http://gebio.com.br/site/wp-content/uploads/2015/05/TCC-Athos-Andrade-Lemos-

Gomes.pdf $>$. Acesso em: 28 mai. 2019.

INVESTSP. Cana-de-açúcar. Disponível em: https://www.investe.sp.gov.br/setores-denegocios/agronegocios/cana-de-acucar/. Acesso em: 19 mai. 2020.

JALLES MACHADO. MEIO AMBIENTE. Disponível em: https://www.jallesmachado.com/pt/meio-ambiente/controle-biologico. Acesso em: 31 ago. 2019.

JORNALCANA. Cana-de-açúcar: Saiba tudo sobre produção de cana-de-açúcar. Disponível em: https://jornalcana.com.br/cana-de-acucar-saiba-tudo-sobre-producao-de-canade-acucar/. Acesso em: 19 mai. 2020.

NOVACANA.COM. A produção de cana-de-açucar no Brasil (e no mundo). Disponível em: https://www.novacana.com/cana/producao-cana-de-acucar-brasil-e-mundo. Acesso em: 18 mai. 2020. 
NUCLEUS. COMPORTAMENTO E CONTROLE DA Diatraea saccharalis NA CULTURA DA CANA-DE-AÇÚCAR. Disponível em: $<$ http://www.nucleus.feituverava.com.br/index.php/nucleus/article/view/311>. Acesso em: 28 mai. 2019.

PARRA, Prof. Dr. José Roberto Postali. Técnicas de criação de insetos para programas de controle biológico: Departamento de Entomologia e Acarologia. Universidade de São Paulo: USP/ESALQ, 2015. $431 \mathrm{p}$.

PORTAL UNAERP. O USO DE AGROTÓXICOS NA CULTURA DE CANA-DEAÇÚCAR E OS PRINCIPAIS RISCOS À SAÚDE DO TRABALHADOR RURAL. Disponível em: https://www.unaerp.br/revista-cientifica-integrada/edicoes-anteriores/volume3-edicao-4/2986-rci-o-uso-de-agrotoxicos-na-cultura-de-cana-de-acucar-e-os-principaisriscos-a-saude-do-trabalhador-rural-06-2018/file. Acesso em: 13 fev. 2020.

REPOSITÓRIO INSTITUCIONAL UFG. INTENSIDADE DE INFESTAÇÃO DE Diatraea saccharalis (FABRICIUS, 1794) (LEPIDOPTERA: CRAMBIDAE) NA QUALIDADE TECNOLÓGICA DE VARIEDADES DE CANA-DE-AÇÚCAR, EM GOIÁS. Disponível em: $\quad<$ https://repositorio.bc.ufg.br/tede/bitstream/tede/4985/5/Dissertação\%20\%20Carlos\%20Alberto\%20da\%20Silva\%20Ferreira\%20-\%202013.pdf>. Acesso em: 02 jun. 2019.

REVISTA FACIDER. O DESENVOLVIMENTO DA CULTURA DA CANA-DEAÇÚCAR NO BRASIL E SUA RELEVÂNCIA NA ECONOMIA NACIONAL. Disponível em: <http://www.sei-cesucol.edu.br/revista/index.php/facider/article/view/37/0>. Acesso em: 27 mai. 2019.

REVISTA PESQUISA FAPESP. José Roberto Postali Parra: Agricultor dos insetos Entomologista defende o uso do controle biológico para combater pragas da lavoura. Disponível em: https://revistapesquisa.fapesp.br/2017/11/24/jose-roberto-postali-parraagricultor-dos-insetos/. Acesso em: 24 ago. 2019.

SCIELO. Reação de cultivares de cana-de-açúcar à broca do colmo. Disponível em: <http:// http://www.scielo.br/pdf/brag/v71n3/aop_1432_12.pdf>. Acesso em: 31 mai. 2019. 